\title{
Inconsistent outcome reporting in large neonatal trials: a systematic review
}

\section{Webbe, JWH}

http://hdl.handle.net/10026.1/17529

10.1136/archdischild-2019-316823

Archives of Disease in Childhood - Fetal and Neonatal Edition

BMJ

All content in PEARL is protected by copyright law. Author manuscripts are made available in accordance with publisher policies. Please cite only the published version using the details provided on the item record or document. In the absence of an open licence (e.g. Creative Commons), permissions for further reuse of content should be sought from the publisher or author. 


\title{
Inconsistent outcome reporting in large neonatal trials: a systematic review
}

\author{
James William Harrison Webbe ${ }^{\circ},{ }^{1}$ Shohaib Ali, ${ }^{2}$ Susanna Sakonidou, ${ }^{1}$ \\ Thomas Webbe, ${ }^{3}$ James M N Duffy, ${ }^{4}$ Ginny Brunton, ${ }^{5}$ Neena Modi (1), \\ Chris Gale ${ }^{0},{ }^{1}$ ' On behalf of the COIN Project Steering Committee
}

\begin{abstract}
- Additional material is published online only. To view please visit the journal online (http://dx.doi.org/10.1136/ archdischild-2019-316823).

${ }^{1}$ Section of Neonatal Medicine, Imperial College London, London, UK

${ }^{2}$ School of Medicine, Imperial College London, London,

London, UK

${ }^{3}$ University of Bristol, Bristol, UK ${ }^{4}$ Nuffield Department of Primary Care Health Sciences, University of Oxford, Oxford, UK

${ }^{5} \mathrm{UCL}$ Institute of Education, University College London, London, UK
\end{abstract}

\section{Correspondence to} Dr James William Harrison Webbe, Academic Neonatal Medicine, Imperial College London, London SW7 2AZ, UK:

j.webbe@imperial.ac.uk

Received 8 January 2019 Revised 17 April 2019

Accepted 24 April 2019 Published Online First

13 May 2019

\begin{abstract}
Objective Inconsistent outcome selection and reporting in clinical trials are important sources of research waste; it is not known how common this problem is in neonatal trials. Our objective was to determine whether large clinical trials involving infants receiving neonatal care report a consistent set of outcomes, how composite outcomes are used and whether parents or former patients were involved in outcome selection.
\end{abstract}

Design A literature search of CENTRAL, CINAHL, EMBASE and MEDLINE was conducted; randomised trials published between 1 July 2012 and 1 July 2017 and involving at least 100 infants in each arm were included. Outcomes and outcome measures were extracted and categorised by physiological system; reported former patient and parent involvement in outcome selection was extracted.

Results Seventy-six trials involving 43126 infants were identified; 216 different outcomes with 889 different outcome measures were reported. Outcome reporting covered all physiological systems but was variable between individual trials: only $67 / 76(88 \%)$ of trials reported survival and 639 outcome measures were only reported in a single trial. Thirty-three composite outcomes were used in 41 trials. No trials reported former patient or parent involvement in outcome selection.

Conclusions Inconsistent outcome reporting and a lack of parent and former patient involvement in outcome selection in neonatal clinical trials limits the ability of such trials to answer clinically meaningful questions. Developing and implementing a core outcome set for future neonatal trials, with input from all stakeholders, should address these issues.

\section{INTRODUCTION}

Neonatal conditions are a leading cause of morbidity and mortality in childhood. Globally 2.7 million babies ${ }^{1}$ die annually in the neonatal period, and in high-income countries approximately 1 in 10 babies will be admitted to a neonatal unit. ${ }^{2}$ Furthermore, neonatal conditions have long-term effects on all physiological systems ${ }^{3}$ that extend into adulthood. ${ }^{4}$ Caring for these babies also has a substantial financial cost: the additional costs incurred during childhood for babies born prematurely in the United Kingdom has been estimated at $£ 3$ billion each year. ${ }^{5}$

Neonatal patients are extremely vulnerable: they often need multiorgan support ${ }^{6}$ and treatments given for one condition can have

\section{What is already known on this topic?}

Problems with outcome selection and reporting are an important cause of research waste in many fields.

- Neonatal conditions and treatments affect outcomes that extend throughout childhood into adult life.

- Neonatal systematic reviews are increasingly unable to identify optimal treatment options due to problems combining evidence from heterogeneous trials.

\section{What this study adds?}

- Neonatal trials report a range of outcomes, using disparate outcome measures, at many different time points.

- We found no evidence of involvement of patients and parents in outcome selection for neonatal trials.

- Developing a neonatal core outcome set will ensure research translates into improvement in neonatal care.

unexpected adverse consequences in other physiological systems. ${ }^{7-10}$ To receive optimal care a robust evidence base is required, so clinicians can make the complex decisions around benefits and risks of different treatments. Unfortunately there is a lack of evidence for many neonatal practices, which leads to variation in both care provision ${ }^{11-13}$ and neonatal outcomes. $^{13-15}$ A review of Cochrane reviews in neonatology found that over $50 \%$ of recent reviews were inconclusive; key factors hindering effective evidence synthesis are heterogeneity of trials and poor methodological quality of studies. ${ }^{16}$

Poor outcome selection, collection and reporting are increasingly recognised as barriers that limit the applicability of research to clinical practice. ${ }^{1718}$ Poorly selected outcomes may make trial findings meaningless to patients or clinicians ${ }^{19}$; poor outcome reporting can cause publication ${ }^{20}$ and reporting bias $^{21}$; and disparate outcome selection can make subsequent meta-analysis impossible. ${ }^{22}$ These problems exist in many other fields ${ }^{23}$; several systematic reviews have shown that heterogeneity of outcome reporting is a particular problem in trials in maternal and newborn health. ${ }^{25-28}$ The use of 
composite outcomes in clinical trials can further contribute to research waste ${ }^{29}$ : individual components within a composite may not be equivalent ${ }^{30}$ and such endpoints may be difficult to interpret in trials where the intervention has opposite directions of effect on different outcomes within a composite. ${ }^{31}$ Some paediatric fields have also found that outcomes are selected to address the needs of researchers ${ }^{32}$ rather than patients and parents. ${ }^{33}$ Public and patient involvement leads to research that is more relevant and useful, ${ }^{34}$ but evidence from other fields indicates that involvement in trial outcome selection is limited, ${ }^{35}$ although this has not been assessed in neonatal trials.

The aims of this review were to determine the range and heterogeneity of outcomes reported in randomised controlled trials of interventions involving infants receiving neonatal care, and whether former patients or parents were involved in the selection of outcomes.

\section{METHODS}

We prospectively registered the study on PROSPERO (Prospective Register of Systematic Reviews): CRD42016042110 ${ }^{36}$ and conducted it in line with Preferred Reporting Items for Systematic Reviews and Meta-Analyses (PRISMA) guidelines ${ }^{37}$ using methods based on previous systematic reviews exploring outcome reporting across randomised trials. ${ }^{25-27}$ We identified studies by searching: Cochrane Central Register of Controlled Trials (CENTRAL); Cumulative Index to Nursing and Allied Health Literature (CINAHL); Excerpta Medica database (EMBASE) and Medical Literature Analysis and Retrieval System Online (MEDLINE). We searched databases from 1997 to July 2017, but due to the large number of studies included the search period was limited from 1 July 2012 to 1 July 2017.

We included studies if they were randomised controlled trials or cluster-randomised trials involving neonates or any infants requiring ongoing care in a neonatal unit beyond the neonatal period; care provided exclusively on postnatal wards or in an outpatient environment was excluded. We last searched the databases on 19 July 2017, and only considered studies in English. The search strategy for CINAHL is included as online supplementary eFigure 1. Three authors (SA, SS, JW) independently double screened potentially relevant records based on titles and abstracts, and reviewed the full text of selected studies to assess eligibility. Due to the high number of trials identified, we only analysed large neonatal trials (defined as over 100 infants in each arm of a study). As many trials lead to more than one publication, we sought out all publications using trial registration records to ensure we had a comprehensive record of the outcomes reported for each trial. To avoid duplication of results if multiple publications related to a single trial met our inclusion criteria, we only included the first paper (with all outcomes coded as above). Three authors (SA, SS and JW) then extracted and categorised outcomes and outcome measures. An 'outcome' was defined as the beneficial or harmful effect a treatment has on an individual while 'outcome measure' was defined as the metric used to characterise this response, in line with Core Outcome Measures in Effectiveness Trials (COMET) initiative guidance. ${ }^{38}$ In the case of discrepancies between the authors during screening or coding, the study was reassessed by three researchers (SA, SS and JW) with input from an additional reviewer (CG) and a majority opinion sought. All screening and coding was undertaken using Eppi-Reviewer 4 software. ${ }^{39}$

We extracted data using a pilot-tested and standardised data extraction form including study characteristics such as trial identifiers, participants and evidence of a protocol/pre-registration.
We systematically extracted all outcomes (eg, bronchopulmonary dysplasia), outcome measures and measurement time points (eg, receiving respiratory support or supplemental oxygen at 28 days) reported in individual clinical trials. We extracted all outcomes reported in the results section or in results tables. If it was clearly stated that outcomes would be measured in the future we also recorded this (particularly if participants are still too young for long-term outcomes to have been reported). We categorised outcomes using a predefined framework of physiological systems ${ }^{40}$ : major systems were respiratory, cardiovascular, gastrointestinal, genitourinary, neurological, infection, pain and neurodevelopmental outcomes; this was developed iteratively as the study progressed. This was used because frameworks from other fields did not relate well to neonatal care ${ }^{4142}$ or missed key concepts. ${ }^{38}$ We also examined the frequency with which predefined neonatal comorbidities are reported in the largest trials. $^{43}$

We extracted data relating to how frequently parents or patients were involved in the choice of outcomes in identified trails from trial publications and protocols where these were available.

We assessed the methodological quality of the included studies using the Jadad criteria. ${ }^{44}$

\section{RESULTS}

Searches identified 24214 records for screening. A total of 76 randomised trials reporting data from 43126 infants met the inclusion criteria (figure 1, online supplementary eTable 1). Fifty six trials (74\%) involved infants born extremely preterm (gestational age at birth $<28$ weeks), 54 trials $(71 \%)$ involved infants born very preterm (gestational age at birth between 28 and 32 weeks) and 25 trials (33\%) involved moderate and late preterm infants (gestational age at birth between 32 and 37 weeks). By contrast only eight trials (11\%) involved term infants. Study quality was good; 72 trials (95\%) scored three or above on the Jadad scale (online supplementary eTable 1).

Across 76 trials 216 distinct outcomes were reported (online supplementary eTable 2). The most commonly reported outcome was survival; reported in 67 trials (88\%). The next most commonly reported outcomes were necrotising enterocolitis (53 trials (70\%)); bronchopulmonary dysplasia (50 trials $(66 \%))$; sepsis (48 trials $(63 \%))$ and retinopathy of prematurity (43 trials (57\%)). In relation to neurodevelopmental outcomes, visual impairment or blindness were only reported in 21 trials $(28 \%)$ and 42 trials $(55 \%)$ did not report any developmental outcomes (online supplementary eTable 3). Even among the 10 trials involving the largest numbers of infants, major neonatal conditions were not universally reported (figure 2). Of the 216 outcomes reported, 92 were only reported in a single trial (figure 3).

Where trials reported the same outcomes, for example, retinopathy of prematurity, these may not be comparable if different outcome measures are used; for example, bilateral retinopathy of prematurity stage $\geq 3$ and retinopathy of prematurity needing surgery (figure 3 ). Sepsis was recorded using 43 different outcome measures (online supplementary eTable 4); bronchopulmonary dysplasia 16 outcome measures and necrotising enterocolitis 13 outcome measures. In relation to the 216 outcomes, 889 different outcomes measures were reported; of these, 639 were only reported in a single trial.

We identified that neonatal trials reported multiple outcome measures, using a number of different time points. The earliest reported outcome was reported $1 \mathrm{~min}$ after birth, ${ }^{45}$ while the 


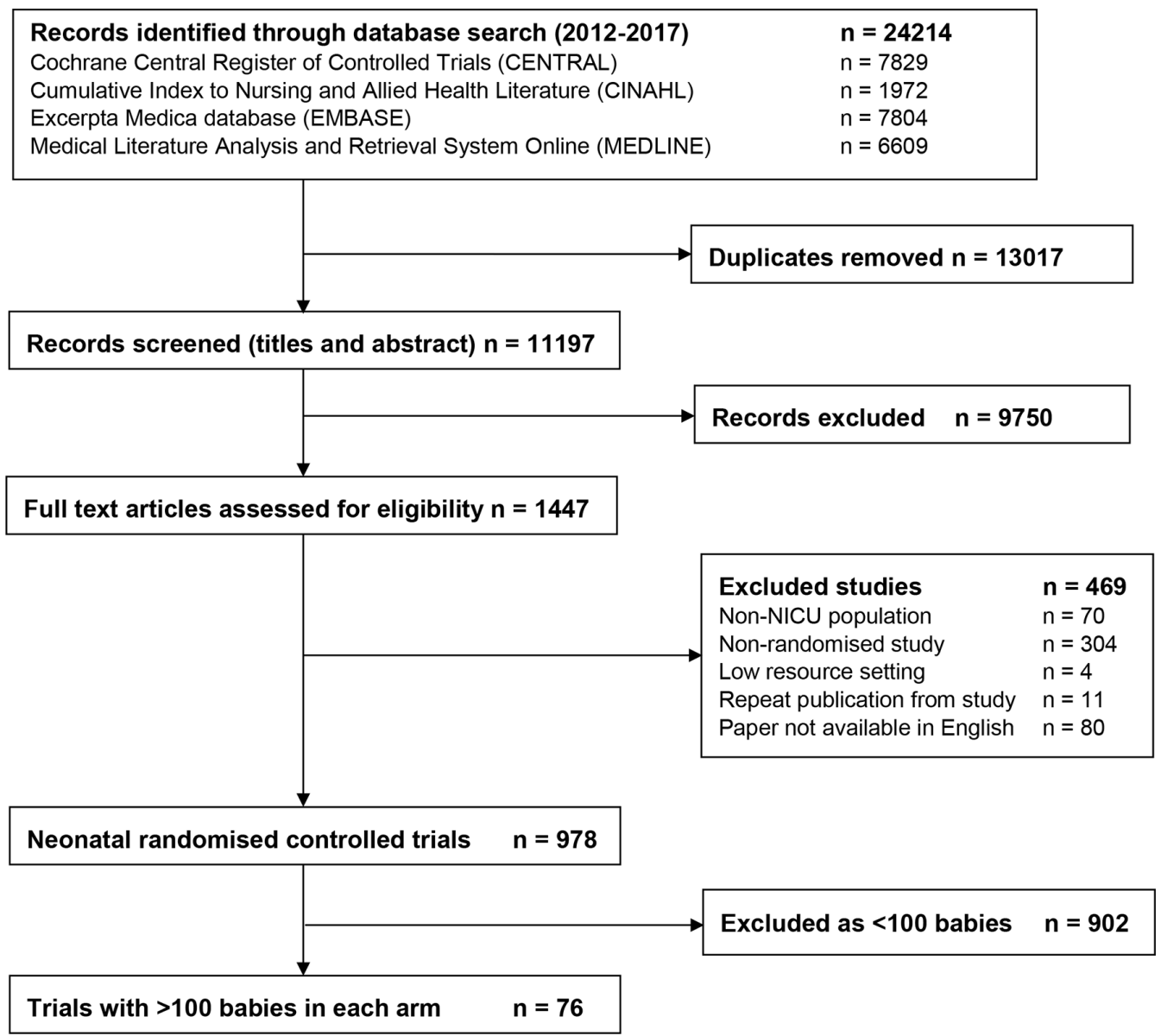

Figure 1 PRISMA flow chart of included papers. NICU, neonatal intensive care unit; PRISMA, Preferred Reporting Items for Systematic Reviews and Meta-Analyses.

latest reported outcomes were reported at 20 years of age. ${ }^{46}$ When considering individual outcomes, survival was reported at 23 different time points (figure 4): of these, 16 related to

Study

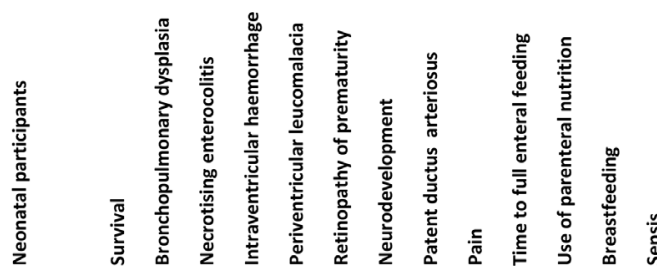

BOOST, $2013 \quad 2448$

Tarnow-Mordi, 20162108

Schmidt, $2012 \quad 2006$

Vaucher, $2012 \quad 1316$

Costeloe, $2016 \quad 1315$

Williams, $2017 \quad 1275$

Collins, 2017

Schmidt, 2013

Jacobs, 2013

Szyld, 2014

Figure 2 ORBIT (Outcome Reporting Bias In Trials) figure illustrating outcome reporting in 10 largest trials. Legend: $\bullet$ Outcome reported in this paper. * Outcome reported in previously published paper relating to this research sample. $\circ$ Outcome documented as planned to be reported in the future. chronological age (ranging from 72 hours of age to 20 years); 3 related to postmenstrual age; 3 to study time points and 1 to discharge from hospital: the most consistently reported was survival to discharge home, reported in 37/76 trials (49\%). We considered the combined impact of outcome measures and time points by looking for a comparable outcome measurement and time point reported consistently across trials (table 1 ).

Composite outcomes were used in 41 trials (54\%); the most commonly reported composite being a composite of death and bronchopulmonary dysplasia (13 trials (17\%)). This composite was reported using six different measures and at two time points. There was heterogeneity among composites: 33 different composite outcomes were reported (online supplementary eTable 5) using 69 incomparable composite outcome measures, with 58 of these outcome measures only reported in a single study.

Among 76 included trials and after reviewing published papers and protocols where available, we found that no trial reported patient or parent involvement in outcome selection.

\section{DISCUSSION}

This review quantifies the range and inconsistency of outcome selection, measurement and reporting in large neonatal trials; this identifies outcome reporting as a major source of research waste in neonatology. There are multiple factors that contribute to this problem: heterogeneous and incomparable outcome measures are used, outcomes are reported at multiple time points (which are frequently poorly specified) and use of composite outcomes 


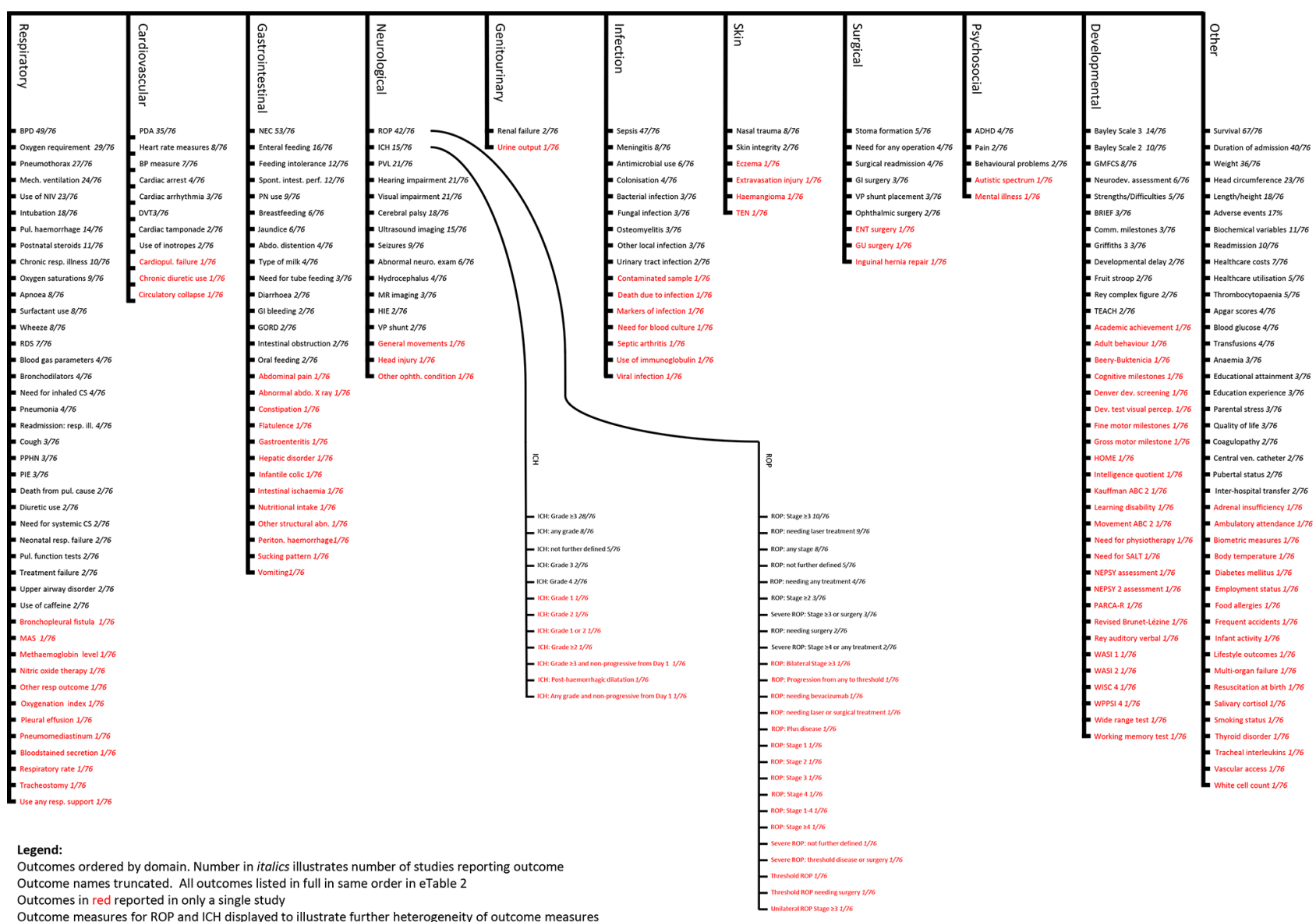

Figure 3 Hierarchical representation of outcomes recorded in 76 large neonatal trials. Legend: Outcomes ordered by domain. Number in italics illustrates number of studies reporting outcome. Outcome names truncated. All outcomes listed in full in same order in online supplementary eTable 2. Outcomes in red reported in only a single study. Outcome measures for retinopathy of prematurity (ROP) and intracranial haemorrhage (ICH) displayed to illustrate further heterogeneity of outcome measures.

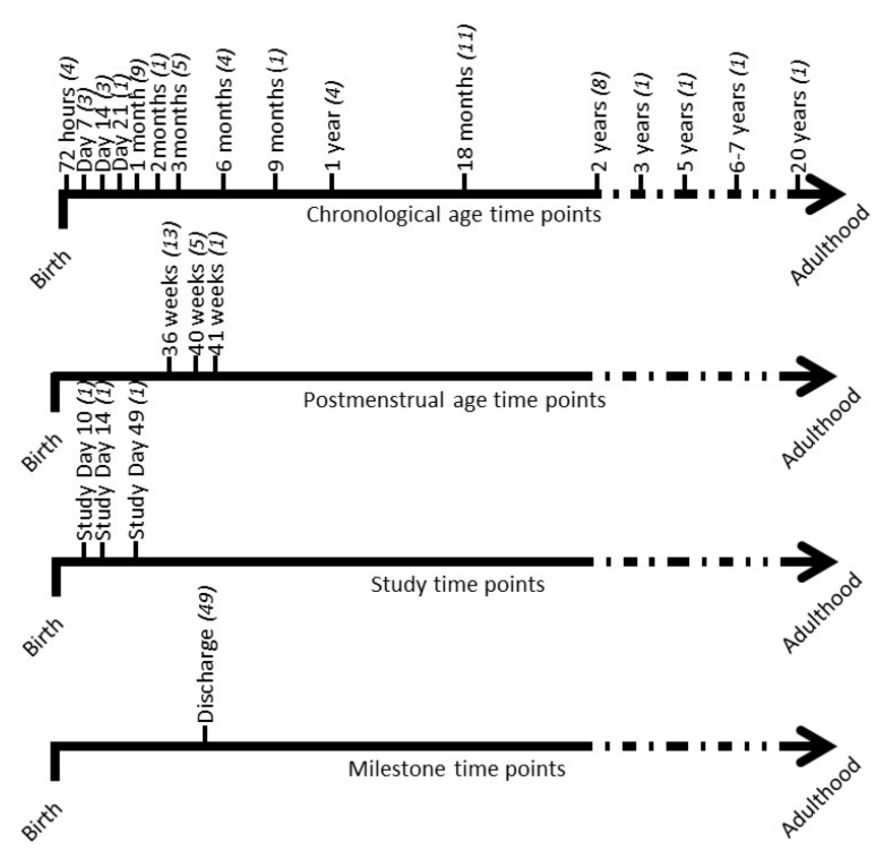

Figure 4 Discrete time points at which survival was measured. Legend: Italicised number in brackets is the percentage of studies that reported survival at each time point. is widespread. Finally, we were unable to identify any reported involvement of parents or patients in outcome selection for the included trials. These problems limit the degree to which results of neonatal clinical trials are able to advance neonatal care.

The strengths of our review are identification of outcomes from a range of international randomised trials, relating to babies of all gestational ages, testing a wide range of interventions. We followed a preregistered protocol $^{36}$ using methods developed in previous similar work ${ }^{25-28}$ and report this review in line with PRISMA guidelines. ${ }^{37}$ Quality of the included trials was generally good; $95 \%$ of trials scored 3 or more on the Jadad scale. ${ }^{44}$ The main limitation of this systematic review is that it is limited to larger neonatal trials (with over 100 infants in each arm). Although a more complete view of the outcomes reported would be obtained by including all trials, the high number of trials identified by our search strategy meant this was unfeasible. Furthermore, our results clearly demonstrate inconsistency and incompleteness of neonatal outcome reporting which would likely be exacerbated by the inclusion of smaller studies. Our review was limited to research conducted in a high-income setting because there are significant differences in practice between high- and low-income settings with distinct fields of research. ${ }^{47}$ Another limitation is that we only included English publications and were only able to assess whether there was reported involvement of patients and parents in outcome selection and did not contact trialists directly. Some trials may have had some input from patients and parents in outcome selection 
Table 1 Comparability of outcome measures and time points for prespecified neonatal outcomes

\begin{tabular}{|c|c|c|c|c|c|}
\hline Outcome & $\begin{array}{l}\text { No of studies } \\
\text { reporting } \\
\text { outcome, n=76 }\end{array}$ & $\begin{array}{l}\text { Most frequently reported } \\
\text { outcome measure }\end{array}$ & $\begin{array}{l}\text { No of studies } \\
\text { reporting outcome } \\
\text { measure, } n=76\end{array}$ & $\begin{array}{l}\text { Most frequently reported } \\
\text { measurement time point }\end{array}$ & $\begin{array}{l}\text { No of studies reporting } \\
\text { comparable outcome } \\
\text { measure and time point, } \\
n=76\end{array}$ \\
\hline Breast feeding & $6(8)$ & $\begin{array}{l}\text { Breast feeding (not further } \\
\text { specified) }\end{array}$ & $3(4)$ & 3 or 6 or 9 or 12 months of age & 2 (3) \\
\hline Bronchopulmonary dysplasia & 49 (64) & Need for oxygen at 36 weeks & $18(24)$ & 36 weeks postmenstrual age & $18(24)$ \\
\hline Enteral feeding & $16(21)$ & $\begin{array}{l}\text { Days to full enteral feeding } \\
\text { (not further specified) }\end{array}$ & $10(13)$ & \multicolumn{2}{|c|}{ Measurement time point not specified for any study* } \\
\hline Intracranial haemorrhage & $36(47)$ & Papile grade $\geq 3$ & $28(37)$ & Discharge home & $5(7)$ \\
\hline Necrotising enterocolitis & $53(70)$ & Bell's stage $\geq 2$ & $18(24)$ & 6 months of age & $4(5)$ \\
\hline Parenteral nutrition (PN) use & $9(12)$ & Duration of PN & $8(11)$ & \multicolumn{2}{|c|}{ Measurement time point not specified for any study* } \\
\hline Patent ductus arteriosus (PDA) & $35(46)$ & PDA needing surgical ligation & $18(24)$ & Discharge home & $4(5)$ \\
\hline $\begin{array}{l}\text { Periventricular } \\
\text { leucomalacia (PVL) }\end{array}$ & $21(28)$ & Cystic PVL & $11(14)$ & 6 months of age & $1(1)$ \\
\hline $\begin{array}{l}\text { Respiratory distress } \\
\text { syndrome (RDS) }\end{array}$ & 7 (9) & RDS (not further specified) & 7 (9) & $\begin{array}{l}10 \text { weeks after start of study } \\
\text { or } 6 \text { months of age }\end{array}$ & $1(1)$ \\
\hline $\begin{array}{l}\text { Retinopathy of } \\
\text { prematurity (ROP) }\end{array}$ & $42(55)$ & ROP stage $\geq 3$ & $10(13)$ & Hospital discharge & 2 (3) \\
\hline Sepsis & $47(62)$ & Late onset sepsis & $6(8)$ & Discharge home & $2(3)$ \\
\hline Survival & 67 (88) & Survival & 67 (88) & Discharge home & $37(49)$ \\
\hline
\end{tabular}

Percentage of studies reporting each outcome/measure/time point is given in italics.

${ }^{*}$ For some outcomes, the reporting time point was not defined for any study.

which was not reported, but as we found no evidence in any of the included trials it seems unlikely such involvement is common or would materially alter these stark findings.

Incomplete reporting of important outcomes has been shown in many fields $s^{49}$ and across women's and newborn health research $2526{ }^{50} 51$; our review demonstrates even an outcome as crucial as survival is not universally reported. Common, important neonatal morbidities like sepsis and necrotising enterocolitis are reported in around two-thirds of papers even though these morbidities are known to be multifactorial ${ }^{5253}$ and may be affected by treatments targeted at other systems. When these outcomes are reported, the range of different time points (23 different time points across 67 trials for survival) and outcome measures (43 different measures across 47 trials for sepsis) makes comparison between studies impossible. Heterogeneity of outcome selection is further illustrated by the large number of outcome measures only reported in a single trial $(639 / 889)$, which mirrors the findings of a review of trials in oncology. ${ }^{54}$

Composite outcomes have been challenged because they may be considered clinically meaningless, ${ }^{19}$ can either inflate effect sizes $^{55} 56$ or mask potentially important effects seen in one component ${ }^{5758}$ and have been explicitly criticised by parents. ${ }^{19}$ This review identifies a further limitation of composite outcomes in neonatal trials, inconsistency in composite construction and reporting: the majority $(58 / 69)$ of composite outcome measures we identified in this review were only used in a single trial. Even when researchers measured similar concepts (eg, death or disability), a new, incomparable measure was often used by individual studies rendering effective meta-analysis of such combined end points impossible.

The high degree of inconsistent outcome selection, measurement and reporting we find has important consequences for evidence-based neonatology, systematic reviews and metaanalyses: the multitude of incomparable outcome measures and time points means that only a small portion of the available evidence can be combined. This is likely to be a key factor behind the increasing number of inconclusive Cochrane reviews in neonatology. ${ }^{16}$ Meta-analyses are essential to identify effective neonatal interventions; they have been instrumental in quantifying the beneficial effect of antenatal steroids ${ }^{59}$ and, more recently, delayed cord clamping in preterm neonates. ${ }^{60}$ However, the scale of the problem identified here suggests that meta-analyses are increasingly unlikely to play such a role in the future unless outcome selection and reporting can be improved.

Public and patient involvement is increasingly recognised to increase the relevance of research to clinical practice and patients' lives. ${ }^{17}{ }^{34}$ Another criticism of clinical trials is that outcome selection reflects the needs of researchers rather than patients or parents, ${ }^{1932}$ and our review supports this critique: we found no evidence of patient or parent involvement in outcome selection. Beyond survival, we found that the most commonly reported outcomes all relate to diagnoses made during the neonatal unit admission; this contrasts with the growing body of evidence that prematurity or sickness in the neonatal period can have effects that last throughout life. ${ }^{46162}$ The correlation between short-term outcomes and longer term difficulties is often inaccurate and imprecise ${ }^{196364}$ and so long-term follow-up is important if trials are to provide evidence on how to optimise outcomes throughout childhood and into adult life. In other fields, patient input has identified important outcomes not previously recognised by researchers. ${ }^{65}{ }^{66}$ This review suggests that more input from patients and parents is needed, particularly in outcome selection as it is known that parents and researchers focus on different neonatal outcomes. ${ }^{67}$

A solution to the problems highlighted in this review, inconsistent outcome reporting and a lack of patient and parent involvement in outcome selection, is the development and application of a core outcome set for neonatal medicine. A core outcome set is a minimum set of outcomes that can be measured in a standardised manner and reported consistently by all trials in a field. ${ }^{38} \mathrm{~A}$ core outcome set is not intended to limit the outcomes recorded by researchers, but rather to specify a minimum set of outcomes, standardised outcome measures and standardised assessment time points. Core outcome sets have been developed in many fields including rheumatology, ${ }^{68}$ paediatric asthma ${ }^{33}$ and 
women's health, ${ }^{69} 70$ and are underpinned by a robust methodology. ${ }^{38}$ The Core Outcomes in Neonatology project is developing a core outcome set for neonatal medicine. ${ }^{43}$

\section{CONCLUSIONS}

There is inconsistency in outcome selection and reporting in clinical trials involving neonates: most trials are missing information on clinically important outcomes. There is no evidence of parent or patient involvement in outcome selection. Developing and implementing a minimum core outcome set for future neonatal trials with input from former patients and parents will address these issues.

Acknowledgements The authors thank the members of the COIN Steering Group for their support, and BLISS and the RCPCH for helping promote this project.

Collaborators Steering Group Chair: Marian Knight (Professor of Maternal and Child Population Health. National Perinatal Epidemiology Unit, Oxford). Project Management Team: Chris GaleClinical (Senior Lecturer in Neonatal Medicine. Imperial College, London) James Webbe (Clinical Research Fellow. Imperial College, London). Steering Group: Elsa Afonso (Neonatal Staff Nurse. Cambridge University Hospitals NHS Foundation Trust, Cambridge); Iyad Al-Muzaffar (Consultant Neonatologist and Parent of preterm baby. Cwm Taf University Health Board, Wales); Ginny Brunton (Midwife and qualitative methodologist. UCL Institute of Education, London); James Duffy (Doctoral Research Fellow in Obstetrics and Gynaecology. Nuffield Department of Primary Health Sciences, Oxford); Anne Greenough (Professor of Neonatology and Clinical Respiratory Physiology. King's College, London); Nigel Hall (Associate Professor of Paediatric Surgery, University of Southampton Jos Latour Professor of Clinical Nursing. Plymouth University, Plymouth); Neil Marlow (Professor of Neonatal Medicine. University College, London); Neena Modi (Professor of Neonatal Medicine. Imperial College, London. Laura Noakes Parent of neonatal patient). Julie Nycyk (Consultant Neonatologist, Sandwell and West Birmingham Hospitals NHS Trust Mehali Patel Research Engagement Officer Bliss, London). Angela Richard-Londt (Parent of neonatal patients). Ben Wills-Eve (Ex-neonatal patient).

Contributors SA, JWHW and CG conceived this systematic review. This protocol was created by SA, GB, JMND and CG. Searches were performed by JWHW. All search results were reviewed by JWHW, SA and SS assessed by the eligibility criteria above. Coding was completed by JWHW, SA and SS. Data analysis was completed by TW and JWHW. The first draft of the manuscript was written by SA and JWHW; CG, JMND and NM edited and reviewed the manuscript. It was approved by JWHW, SA, SS, TW, GB, JMND, CG, NM and the COIN Steering Group.

Funding This research is sponsored by Imperial College London and supported by an MRC Clinician Scientist Fellowship award to CG (MR/N008405/1) and salary support for JWHW from the Portland Hospital. Imperial College London, the MRC and the Portland Hospital had no involvement in the research or this publication.

Competing interests $C G$ is part of an international team developing reporting guidance (a CONSORT extension) for clinical trials using cohorts and routinely collected health data. He has received support from Chiesi Pharmaceuticals to attend an educational conference; in the past 5 years, he had been the investigator on received research grants from Medical Research Council, National Institute of Health Research, Canadian Institute of Health Research, Department of Health in England, Mason Medical Research Foundation, and Westminster Medical School Research Trust and Chiesi Pharmaceuticals; JW has received support from Chiesi Pharmaceuticals to attend an educational conference and has received a research grant from Mason Medical Research Foundation.

Patient consent for publication Not required.

Provenance and peer review Not commissioned; externally peer reviewed.

Data sharing statement No unpublished data are available for this study.

ORCID iDs

James William Harrison Webbe http://orcid.org/0000-0001-8546-3212

Neena Modi http://orcid.org/0000-0002-2093-0681

Chris Gale http://orcid.org/0000-0003-0707-876X

\section{REFERENCES}

1 UNICEF, WHO, World Bank. UN-DESA Population Division. Levels and trends in child mortality 2015, 2016

2 Royal College of Paediatrics and Child Health. National Neonatal Audit Programme 2016 Annual Report on 2015 data, 2016.

3 Saigal S, Doyle LW. An overview of mortality and sequelae of preterm birth from infancy to adulthood. Lancet 2008;371:261-9.
4 Saigal S, Day KL, Van Lieshout RJ, et al. Health, Wealth, Social Integration, and Sexuality of Extremely Low-Birth-Weight Prematurely Born Adults in the Fourth Decade of Life. JAMA Pediatr 2016;170:678-86.

5 Mangham LJ, Petrou S, Doyle LW, et al. The cost of preterm birth throughout childhood in England and Wales. Pediatrics 2009;123:e312-27.

6 Glass HC, Costarino AT, Stayer SA, et al. Outcomes for extremely premature infants. Anesth Analg 2015;120:1337-51.

7 Lanman JT, Guy LP, Dancis J. Retrolental fibroplasia and oxygen therapy. J Am Med Assoc 1954;155:223-6.

8 Barrington KJ. The adverse neuro-developmental effects of postnatal steroids in the preterm infant: a systematic review of RCTs. BMC Pediatr 2001;1:1.

9 Aziz K. Postnatal steroids for chronic lung disease - a lesson learned. Paediatr Child Health 2002;7:9-10.

10 SUPPORT Study Group of the Eunice Kennedy Shriver NICHD Neonatal Research Network. Target Ranges of Oxygen Saturation in Extremely Preterm Infants. $N$ Eng/ J Med Overseas Ed 2010;362:1959-69.

11 Klingenberg C, Embleton ND, Jacobs SE, et al. Enteral feeding practices in very preterm infants: an international survey. Arch Dis Child Fetal Neonatal Ed 2012:97:F56-61.

12 Lapillonne A, Carnielli VP, Embleton ND, et al. Quality of newborn care: adherence to guidelines for parenteral nutrition in preterm infants in four European countries. BMJ Open 2013;3:e003478.

13 Rysavy MA, Li L, Bell EF, et al. Between-hospital variation in treatment and outcomes in extremely preterm infants. N Engl J Med 2015;372:1801-11.

14 Shah PS, Lui K, Sjörs G, et al. Neonatal Outcomes of Very Low Birth Weight and Very Preterm Neonates: An International Comparison. J Pediatr 2016:177:144-52.

15 Helenius K, Sjörs G, Shah PS, et al. Survival in Very Preterm Infants: An International Comparison of 10 National Neonatal Networks. Pediatrics 2017;140:e20171264-9.

16 Willhelm C, Girisch W, Gottschling S, et al. Systematic Cochrane reviews in neonatology: a critical appraisal. Pediatr Neonatol 2013;54:261-6.

17 Heneghan C, Goldacre B, Mahtani KR. Why clinical trial outcomes fail to translate into benefits for patients. Trials 2017;18:122.

18 Duffy JMN, Ziebland S, von Dadelszen P, et al. Tackling poorly selected, collected, and reported outcomes in obstetrics and gynecology research. Am J Obstet Gynecol 2019;220:71.e1-71.e4

19 Janvier A, Farlow B, Baardsnes J, et al. Measuring and communicating meaningful outcomes in neonatology: A family perspective. Semin Perinatol 2016;40:571-7.

20 Schmucker C, Schell LK, Portalupi S, et al. Extent of non-publication in cohorts of studies approved by research ethics committees or included in trial registries. PLOS One 2014:9:e114023.

21 Kirkham JJ, Dwan KM, Altman DG, et al. The impact of outcome reporting bias in randomised controlled trials on a cohort of systematic reviews. BMJ 2010;340:C365.

22 Bender R, Bunce C, Clarke M, et al. Attention should be given to multiplicity issues in systematic reviews. J Clin Epidemiol 2008;61:857-65.

23 Johnston BC, Shamseer L, da Costa BR, et al. Measurement issues in trials of pediatric acute diarrheal diseases: a systematic review. Pediatrics 2010;126:e222-31.

24 Chong LSH, Sautenet B, Tong A, et al. Range and Heterogeneity of Outcomes in Randomized Trials of Pediatric Chronic Kidney Disease. J Pediatr 2017;186:110-7. e11.

25 Duffy J, Hirsch M, Kawsar A, et al. Outcome reporting across randomised controlled trials evaluating therapeutic interventions for pre-eclampsia. BJOG 2017;124:1829-39.

26 Perry H, Duffy JMN, Umadia $\mathrm{O}$, et al. Outcome reporting across randomized trials and observational studies evaluating treatments for twin-twin transfusion syndrome: systematic review. Ultrasound Obstet Gynecol 2018;52:577-85.

27 Hirsch M, Duffy JMN, Kusznir JO, et al. Variation in outcome reporting in endometriosis trials: a systematic review. Am J Obstet Gynecol 2016;214:452-64.

28 Duffy JMN, Hirsch M, Gale C, et al. A systematic review of primary outcomes and outcome measure reporting in randomized trials evaluating treatments for preeclampsia. Int J Gynaecol Obstet 2017;139:262-7.

29 Freemantle N, Calvert M, Wood J, et al. Composite outcomes in randomized trials: greater precision but with greater uncertainty? JAMA 2003;289:2554-9.

30 Warren JB. Composite endpoints and the distortion of risk-benefit analysis. $\mathrm{Br} J \mathrm{Clin}$ Pharmacol 2017:83:221-3.

31 Carlo WA, Finer NN, Walsh MC, et al. Target ranges of oxygen saturation in extremely preterm infants. N Engl J Med 2010;362:1959-69.

32 Sinha IP, Williamson PR, Smyth RL. Outcomes in clinical trials of inhaled corticosteroid for children with asthma are narrowly focussed on short term disease activity. PLoS One 2009:4:e6276.

33 Sinha IP, Gallagher R, Williamson PR, et al. Development of a core outcome set for clinical trials in childhood asthma: a survey of clinicians, parents, and young people. Trials 2012;13:103.

34 Staley K. Exploring Impact: Public involvement in NHS, public health and social care research. Eastleigh: INVOLVE, 2009.

35 Kirwan JR, Hewlett SE, Heiberg T, et al. Incorporating the patient perspective into outcome assessment in rheumatoid arthritis--progress at OMERACT 7. J Rheumatol 2005;32:2250-6 
36 PROSPERO database. http://www.crd.york.ac.uk/prospero/display_record.asp?ID= CRD42016042110 (14 Nov 2018).

37 Moher D, Liberati A, Tetzlaff J, et al. PRISMA Group. Preferred reporting items for systematic reviews and meta-analyses: the PRISMA statement. Ann Intern Med 2009;151:264-9.

38 Williamson PR, Altman DG, Bagley H, et al. The COMET Handbook: version 1.0. Trials 2017;18:18.

39 Thomas J, Brunton J, Graziosi S. EPPI-Reviewer 4: software for research synthesis. EPPI-Centre Software. London: Social Science Research Unit, Institute of Education, University of London, 2010.

40 NHS Digital. National Neonatal Data Set. 2017 http://www.datadictionary.nhs.uk/data dictionary/messages/dinical_data_sets/data_sets/national_neonatal_data_set/national_ neonatal_data_set_-_episodic_and_daily_care_fr.asp?shownav=1 (12 Feb 2017).

41 Carpenter WR, Meyer AM, Abernethy AP, et al. A framework for understanding cance comparative effectiveness research data needs. J Clin Epidemiol 2012:65:1150-8.

42 Bakas T, McLennon SM, Carpenter JS, et al. Systematic review of health-related quality of life models. Health Qual Life Outcomes 2012:10:134.

43 Webbe J, Brunton G, Ali S, et al. Developing, implementing and disseminating a core outcome set for neonatal medicine. BMJ Paediatr Open 2017:1:e000048.

44 Jadad AR, Moore RA, Carroll D, et al. Assessing the quality of reports of randomized clinical trials: is blinding necessary? Control Clin Trials 1996;17:1-12.

45 Szyld E, Aguilar A, Musante GA, et al. Comparison of devices for newborn ventilation in the delivery room. J Pediatr 2014;165:234-9.

46 Charpak N, Tessier R, Ruiz JG, et al. Twenty-year Follow-up of Kangaroo Mother Care Versus Traditional Care. Pediatrics 2017;139:e20162063.

47 Althabe F, Belizán JM, McClure EM, et al. A population-based, multifaceted strategy to implement antenatal corticosteroid treatment versus standard care for the reduction of neonatal mortality due to preterm birth in low-income and middle-income countries: the ACT cluster-randomised trial. Lancet 2015;385:629-39.

48 Allin BS, Irvine A, Patni N, et al. Variability of outcome reporting in Hirschsprung's Disease and gastroschisis: a systematic review. Sci Rep 2016;6:38969.

49 Kirkham JJ, Gargon E, Clarke M, et al. Can a core outcome set improve the quality of systematic reviews? - a survey of the Co-ordinating Editors of Cochrane review groups. Trials 2013;14:14.

50 Pergialiotis V, Durnea C, Elfituri A, et al. Do we need a core outcome set for childbirth perineal trauma research? A systematic review of outcome reporting in randomised trials evaluating the management of childbirth trauma. BJOG 2018;125:1522-31.

51 de Mattos Lourenco TR, Pergialiotis V, Duffy JMN, et al. A systematic review on reporting outcomes and outcome measures in trials on synthetic mesh procedures for pelvic organ prolapse: Urgent action is needed to improve quality of research. Neurourol Urodyn 2019;38:509-24.
52 Schnabl KL, Van Aerde JE, Thomson AB, et al. Necrotizing enterocolitis: a multifactorial disease with no cure. World J Gastroenterol 2008;14:2142-61.

53 Wolach B. Neonatal sepsis: pathogenesis and supportive therapy. Semin Perinatol 1997;21:28-38.

54 Hirsch BR, Califf RM, Cheng SK, et al. Characteristics of oncology clinical trials: insights from a systematic analysis of ClinicalTrials.gov. JAMA Intern Med 2013;173:972-9.

55 Cordoba G, Schwartz L, Woloshin S, et al. Definition, reporting, and interpretation of composite outcomes in clinical trials: systematic review. BMJ 2010;341:c3920.

56 Barrington K. Number needed to suffer? Acta Paediatr 2018;107:203.

57 Tarnow-Mordi WO, Darlow B, Doyle L. Target ranges of oxygen saturation in extremely preterm infants. N Engl J Med 2010;363:1285. author reply -6.

58 Tarnow-Mordi W, Morris J, Kirby A, et al. Delayed versus Immediate Cord Clamping in Preterm Infants. N Engl J Med 2017;377:2445-55.

59 Crowley PA. Antenatal corticosteroid therapy: a meta-analysis of the randomized trials, 1972 to 1994. Am J Obstet Gynecol 1995;173:322-35.

60 Fogarty M, Osborn DA, Askie L, et al. Delayed vs early umbilical cord clamping for preterm infants: a systematic review and meta-analysis. Am J Obstet Gynecol 2018:218:1-18.

61 Doyle LW, Anderson PJ. Adult outcome of extremely preterm infants. Pediatrics 2010;126:342-51

62 Roberts G, Cheong JL. Long-term growth and general health for the tiniest or most immature infants. Semin Fetal Neonatal Med 2014;19:118-24.

63 Edwards AD, Redshaw ME, Kennea N, et al. Effect of MRI on preterm infants and their families: a randomised trial with nested diagnostic and economic evaluation. Arch Dis Child Fetal Neonatal Ed 2018;103:F15-21.

64 Clemm H, Røksund O, Thorsen E, et al. Aerobic capacity and exercise performance in young people born extremely preterm. Pediatrics 2012;129:e97-105.

65 de Wit M, Abma T, Koelewijn-van Loon M, et al. Involving patient research partners has a significant impact on outcomes research: a responsive evaluation of the international OMERACT conferences. BMJ Open 2013;3:e002241.

66 Arnold LM, Crofford LJ, Mease PJ, et al. Patient perspectives on the impact of fibromyalgia. Patient Educ Couns 2008;73:114-20.

67 Webbe J, Brunton G, Ali S, et al. Parent, patient and clinician perceptions of outcomes during and following neonatal care: a systematic review of qualitative research. BMJ Paediatr Open 2018;2:e000343.

68 Tugwell P, Boers M, Brooks P, et al. OMERACT: an international initiative to improve outcome measurement in rheumatology. Trials 2007;8:8.

69 Duffy J, Rolph R, Gale C, et al. Core outcome sets in women's and newborn health: a systematic review. BJOG 2017;124:1481-9.

70 van 't Hooft J, Duffy JM, Daly M, et al. A Core Outcome Set for Evaluation of Interventions to Prevent Preterm Birth. Obstet Gynecol 2016;127:49-58. 